Military Technical College Kobry El-Kobbah, Cairo, Egypt

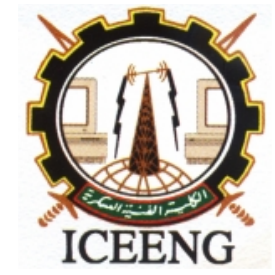

\author{
$8^{\text {th }}$ International Conference \\ on Electrical Engineering \\ ICEENG 2012
}

\title{
Single and Combined Electromagnetic Wave Propagation Coverage of VOR Stations in Irregular Terrain Air ports
}

By
M. A. Abdalla*
A. M. Mahrous*
A. Helmy*

\begin{abstract}
:
In this paper, the electromagnetic propagation coverage of different VOR stations over irregular terrain airport locations has been presented. Three different investigation studies for different airports in the heavy terrain red sea area are introduced. The calculation has been done applying Longley rice propagation model. The theoretical calculation concepts of the employed electromagnetic propagation model are discussed. The coverage areas for the two studied air ports were plotted using digital Two dimensional geographical maps with 90 meter resolution. The results are compared to standard line of sight model.
\end{abstract}

\section{Keywords:}

Electromagnetic waves, Propagation model, Coverage area, Longley Rice

* Egyptian Armed Forces

\section{Introduction:}

The continuous and increasing growth of wireless communication systems introduces new challenge for the good service planning. Different planning parameters can be investigated such as frequency, polarization, and coverage area. The good coverage area 
prediction can help designer for good examination of the service area for a certain applications. Also, it helps the designer in assigning different users to make use of the communication services. The increasing demand for using propagation models encourages researchers for modifying and applying different propagation model calculation and coverage area simulation on the different communication environment. Propagation models are classified into deterministic [1-6] and empirical [7-16] models. Deterministic models introduces more accurate path loss calculation and they are applied mainly in the low coverage distance applications at which building dimensions are major factor in estimating the coverage area. On the other hand, the empirical models are more suitable for large distance communications. Several propagation models have been used for the prediction of coverage area. The two popular propagation models approved by the ITU are the Longley Rice model [7-10] and Okumura-Hata model [11-16]. The different propagation models are mainly working for accurate computation of the path gain between the transmitter and receiver according to the employed communication service. However, some skill is needed in choosing the optimum model for certain communication case. The Okumura-Hata method is not believed to be valid below $150 \mathrm{MHz}$ while the Longley Rice model is nominally valid in the range $20 \mathrm{MHz}$ to $20 \mathrm{GHz}$ and for path lengths between $1 \mathrm{~km}$ and $2000 \mathrm{~km}$ [17].

The proper estimation of the application service area should take into account a trade off between the economic and service quality point of view. The efficient use of the application electromagnetic coverage area requires the knowledge of several technical parameters which are based partly on equipment performance considerations, the geographical area around the transmitter and receiver, and mainly on the effects of the propagation medium on the information transmitted between two points on or near the earth's surface

In this paper we present the calculation of very high frequency (VHF) electromagnetic wave propagation over a heavy terrain land at low altitudes. The calculation for the propagation model was based on the Langley-Rice model. The study objective is satisfied through a comparison study between the employed coded propagation model and conventional line of sight calculation.

The system employed in this study as a transmitter was selected as aircraft navigation aid which is a very high frequency omnidirectional radio (VOR) station located in different heavy terrain airport at Red sea area. VOR is a main short-range navigational aid operates at the VHF band. Short range infers that ranges up to $370 \mathrm{~km}$. Our work was focused on the calculation of the coverage area of this system on approaching and landing process. Therefore, the employed receiver is was an approaching aircraft at low altitudes. Two studied altitudes of 100 meter and 300 meter was investigated for each studied airport case. The results convey that the employed propagation model is capable of predicting path loss if the terrain profile between the transmitter and receiver. Detailed analysis reports for the studied case are extracted. 


\section{Propagation Mechanism:}

\section{A General. Propagation Mechanism:}

The electromagnetic wave propagation can be due to the direct electromagnetic wave propagation, reflection, diffraction, and scattering waves. Direct wave is due to the line of sight (LOS) communication between the transmitter and receiver. It is only affected by the earth curvature and it is independent on the terrains. The other two factors are heavily affected by the terrain between the transmitter and receiver. Reflection happens due to the incidence of the propagated electromagnetic waves on any other surface between the transmitter and receiver. Reflection can be simply from the ground surface separating the transmitter and receiver. Also, it can be due to presence of surrounding terrains whose dimensions are very large compared the incident electromagnetic wave wavelength. Reflection causes the electromagnetic wave to be traveling in the opposite direction. Hence, the reflected waves and the direct one can interfere with the direct wave in either constructive or destructive way at the receiver antenna input.

Diffraction occurs when the travelling electromagnetic wave encounters an obstacle that cannot penetrate it. Diffraction may occur due to effect the electromagnetic wave scattering from channel objects. Mainly, these objects have dimensions are less than the electromagnetic wavelength travelling through the channel. Both diffraction and scattering, causes transmitted electromagnetic waves to be re-directed into many different directions. Therefore, they can be accounted for the main reason for achieving communication behind LOS range; i.e without a LOS path. This phenomenon is called "shadowing," since the diffracted field can reach a receiver even when it is shadowed by an obstruction; i.e behind LOS. In general, the attenuation over the path between the transmitting and receiving antennas can be described as

$$
\boldsymbol{P}_{\boldsymbol{r}}=\boldsymbol{P}_{\boldsymbol{t}}+\boldsymbol{G}_{\boldsymbol{t}}+\boldsymbol{G}_{\boldsymbol{r}}-\boldsymbol{L}_{\boldsymbol{f}}(\mathrm{dBm})
$$

where $P_{t}, P_{r}, G_{t}$, and $G_{r}$ are the transmitted power, received power, transmitting antenna gain, and receiving antenna gain, respectively, whereas $\mathrm{L}_{\mathrm{fs}}$ is the free space attenuation (free-space transmission loss) in $\mathrm{dB}$. Considering all possible propagation mechanism is the free space loss can be calculated. The main concept of establishing the propagation model computation is to build up the reflected and refracted rays at each point at which an obstacle in the path between transmitter and receiver is found [18].

The simplest propagation model is the free space model that assumes the most perfect condition; propagation happens in a free empty space without any kind of obstacles, reflectors, edges, or even air particles. This model accounts for basic free-space loss factor which is the loss due to spherical spreading of the energy by the antenna. Another propagation model is the two-ray model which accounts for the reflection from the spherical curvature ground, and supposes that the signal strength at the receiver results from superposition of direct reflected signals. This model is more practical model but it still does not consider the diffraction and scattering propagation mechanisms. 


\section{B-Longley-Rice Model Propagation Mechanism:}

In this section, we explain the mathematical concept of the employed Longley-Rice propagation model. Longley-Rice propagation model, also known as "Irregular Terrain Model" (ITM)), estimates radio propagation losses over irregular terrain in the range 20 $\mathrm{MHz}$ to $20 \mathrm{GHz}$ frequencies as a function of distance and the variability of signal in time and space [7-10]. Computing of signal loss is based on electromagnetic theory and on statistical analyses. It has been complemented by empirical dependences, during tests and measurements. Several methodology techniques were introduced for the standardization of the Longley Rice model in VHF.UHF bands [19-21].

In the VHF/UHF band electromagnetic propagation, terrains such as mountain edges are sufficiently large compared to a wavelength to become efficient reflectors. Thus, by applying the Longley Rice model, it has been confirmed that terrain scattering can allow communications beyond the radio line of sight. Also, the ITM model is capable for both VHF/UHF broadcast and land mobile systems whose propagation mechanism is quite similar. Both services have fixed communication end and arbitrary positioned communication end. The common elements for both types of services, which distinguish them from point-to-point communication links, are 1) the arbitrariness of at least one of the link terminal locations, and 2) the impracticality of using deterministic methods for defining transmission loss over irregular terrain in such cases. The ITM calculation algorithm based on different input parameters can be divided into deterministic parameters and statistical parameters.

The deterministic parameters are:

- System input parameters: frequency, antenna heights, antenna effective heights, distance between two terminals, distance from each terminal to its radio horizon, and polarization.

- Environmental parameters: full terrain data (terrain irregularity), and the path coordinates, electrical ground conductivity, relative permittivity, and refractivity.

The Statistical parameters:

- Time variability, location variability, and situation variability

- Climate, reliability, confidence levels, percent time.

- Some sitting criteria that describe the care taken at each terminal to assure good radio propagation conditions. This is expressed in most algorithms as Two discrete inputs, random, with care, and with great care.

It should be noted that these inputs are calculated from empirical formulas in the prediction area. 


\section{ITM Algorithm Outputs:}

The main goal of ITM model, or simply any propagation model, is to calculate the path loss (attenuation). Two types of attenuations (outputs) can be calculated through the model according to the designers well. Those are:

- Reference attenuation which is the median attenuation relative to free space signal.

- The second output is three dimensional cumulative distribution of attenuation in which time, location, and situation variability are all accounted for $A\left(q_{T}, q_{L}, q_{S}\right)$. This can be said as for $q_{3}$ of the situations there will be at least $q_{L}$ of locations where the attenuation doesn't exceed $A\left(q_{T}, q_{L}, q_{S}\right)$. for at least $q_{T}$ of the time.

\section{ITM Algorithm}

- Preparatory calculations for geometrical parameters:

The parameters $h_{e j}$ (Antenna effective height), $d_{L j}$ (Distance from the antenna to its radio horizon), $\theta_{e j}$ (Elevation angle to the radio horizon from the antenna height),$j$ $=1,2$, which are part of the input in the point-to-point mode are, in the area prediction mode, estimated using empirical formulas in which $\Delta h$ (Terrain irregularity parameter) plays an important role.

Antenna effective height:

$h_{e j}=h_{g j} \quad$ If terminal $j$ is sited at random.

$h_{\theta j}=h_{g j}+B_{j}^{\prime} e^{-2 h_{g j} / \Delta h} \quad$ If terminal $j$ is not sited at random.

$B_{j}^{\prime}=\left(B_{j}-H_{1}\right) \sin \left(\frac{\pi}{2} \min \left(\frac{h_{g 1}}{H_{2}}, 1\right)\right)+H_{1} \quad$ with $H_{1}=1 m, H_{2}=5 m$,

$B_{j}=\left\{\begin{array}{lr}5 \mathrm{~m} & \text { if terminal } j \text { is sited with care } \\ 10 \mathrm{~m} & \text { if terminal } j \text { is sited with great care }\end{array}\right.$

Distance to horizon:

$$
\begin{aligned}
& d_{L s j}=\sqrt{2 \frac{h_{e j}}{\gamma_{\theta}}}
\end{aligned}
$$

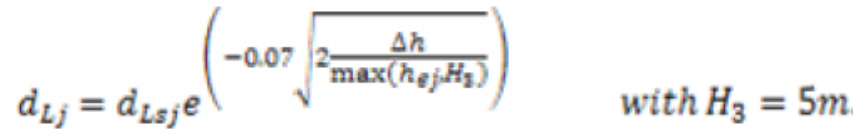


Elevation angle:

$\theta_{e j}=\frac{\left[0.65 \Delta h\left(\frac{d_{L s j}}{d_{L j}}-1\right)-2 h_{e j}\right]}{d_{L s j}}$

Calculation for both modes:

$$
\begin{aligned}
& d_{L s j}=\sqrt{2 h_{e j} / \gamma_{e}}, \quad j=1,2 \\
& d_{L s}=d_{L s 1}+d_{L s 2} \\
& d_{L}=d_{L 1}+d_{L 2} \\
& \theta_{e}=\max \left(\theta_{e 1}+\theta_{e 2},-d_{L} \gamma_{e}\right) \\
& \Delta h(s)=\left(1-0.8 e^{-s / D}\right) \Delta h \quad \text { with } D=50 \mathrm{~km} . \\
& \sigma_{h}(s)=0.78 \Delta h(s) e^{-\sqrt[4]{\Delta h(s) / H}} \quad \text { with } H=16 \mathrm{~m} .
\end{aligned}
$$

- Reference attenuation calculations:

The reference attenuation is determined as a function of the distance $\mathrm{d}$ from the piecewise formula

$$
A_{r e f}=\left\{\begin{array}{lr}
\max \left(0, A_{e l}+K_{1} d+K_{2} \ln \left(\frac{d}{d_{L s}}\right)\right) & d \leq d_{L s} \\
A_{e d}+m_{d} d & d_{L s} \leq d \leq d_{x} \\
A_{e s}+m_{s} d & d_{x} \leq d
\end{array}\right.
$$

where the coefficients Ael, K1, K2, Aed, md, Aes, ms, and the distance dx are calculated using the algorithms below. The three intervals de_ned here are called the line-of-sight, diffraction, and scatter regions, respectively.

\section{- Variability of attenuation:}

This would be the final step in the ITM algorithm. In this step we want to compute $A\left(q_{T}, q_{L}, q_{S}\right)$ where $q_{T}, q_{L}, q_{S}$, are the desired fractions of time, locations, and situations, respectively. In the point-to-point mode, we would want a two-fold quantile 
$A\left(q_{T}, q_{S}\right)$, but in the present model this is done simply by computing the three-fold quantile with $q_{L}$ equal to 0.5 . At the end, the cumulative attenuation $A\left(z_{T}, z_{L}, z_{S}\right)$, which is function of the deviations $z_{T}, z_{L}, z_{S}$ that are function of time, location, and situation variability, is to be calculated from the following:

$$
\begin{aligned}
& A\left(z_{T}, z_{L}, z_{S}\right)=\left\{\begin{array}{lr}
A^{\prime} & A^{\prime} \geq 0 \\
A^{\prime} \frac{29-A^{\prime}}{29-10 A^{\prime}} & \text { otherwisu }
\end{array}\right. \\
& A^{\prime}=A_{\text {ref }}-V_{\text {med }}-Y_{T}-Y_{L}-Y_{S}
\end{aligned}
$$

where the adjustment $V_{\text {med }}$ and the deviations $Y_{T}, Y_{L}$, and $Y_{S}$ are to be calculated accounting for time, location, and situation variability through a statistical analysis that builds up the algorithm.

By calculating the attenuation has been calculated throughout the algorithm, the received power at each point can be predicted using (1) and compared to the receiver sensitivity to decide if this point is within the coverage area or not. It is worth to mention that this calculation requires 3D digital maps with all geographical data to apply the previous algorithm at each point. This will be applied in the next section for two different locations with different irregular terrains.

\section{Results and Discussion:}

The problem addressed in this paper is focused into achieving confirmed service for VOR base stations serving approaching planes at different altitudes. For proper system operation, the RF received signal power must be maintained above a certain level. In our work, this level was selected to be the receiver sensitivity. The study was for medium terrain surrounding airport (International Sharm El-shekh airport) and heavy terrain airport (St. Katherine airport). All studied VOR systems was assumed operating in the VHF band at frequencies 100-150 MHz.

The system parameters for each studied case are as follow: The ground station parameters, transmitting power $=50$ watt, sensitivity $=-107 \mathrm{dBm}$, antenna pattern is omnidirectional, antenna gain $=2 \mathrm{~dB}$, antenna height $=15 \mathrm{~m}$, additional loss $=4 \mathrm{~dB}$. The communication system in the moving plane has the following parameters, transmitting power $=10$ watt, sensitivity $=-99 \mathrm{dBm}$, antenna pattern is omnidirectional, antenna gain $=-1 \mathrm{~dB}$, antenna height $=100$ meter, and 300 meter, additional loss $=0 \mathrm{~dB}$.

In all studied cases, the results were computed using Longley rice model and compared with free space model. The free space model coverage radius is calculated in terms of transmitting and receiving antenna heights as follow:

$$
\boldsymbol{d}_{\boldsymbol{0}}=4.12\left(\sqrt{\boldsymbol{h}_{1}}+\sqrt{\boldsymbol{h}_{2}}\right) \quad(\boldsymbol{k m})
$$


For the case of aircraft at 100 meter height, this range is $57 \mathrm{~km}$ and it is $87 \mathrm{~km}$ for 300 meter aircraft height.

\section{A-Medium Terrain Airport Results and Discussions}

The 3D surrounding of Sharm El-shekh airport is shown in Figure 1. As shown, apart of the south direction, there are irregular terrains every where around the airport. However the other side of the airport is mainly surrounded by red sea. Within the VHF band, such terrain irregular can affect the electromagnetic propagation. The coverage area has been calculated for 100 meter and 300 meter height and plotted in Figure 2 and Figure 3, respectively. As shown in the two figures, the coverage is irregular distribution. However, the coverage area is almost uniform within the south zone within which no irregular terrain is founded. The contribution of the computed coverage area was investigated by comparing it to that area computed by free space model whose result is a simple circle of constant radius. These two results are plotted in Figure 2, and Figure 3, respectively, along with the Longley Rice model computed results for the sake of results illustration.

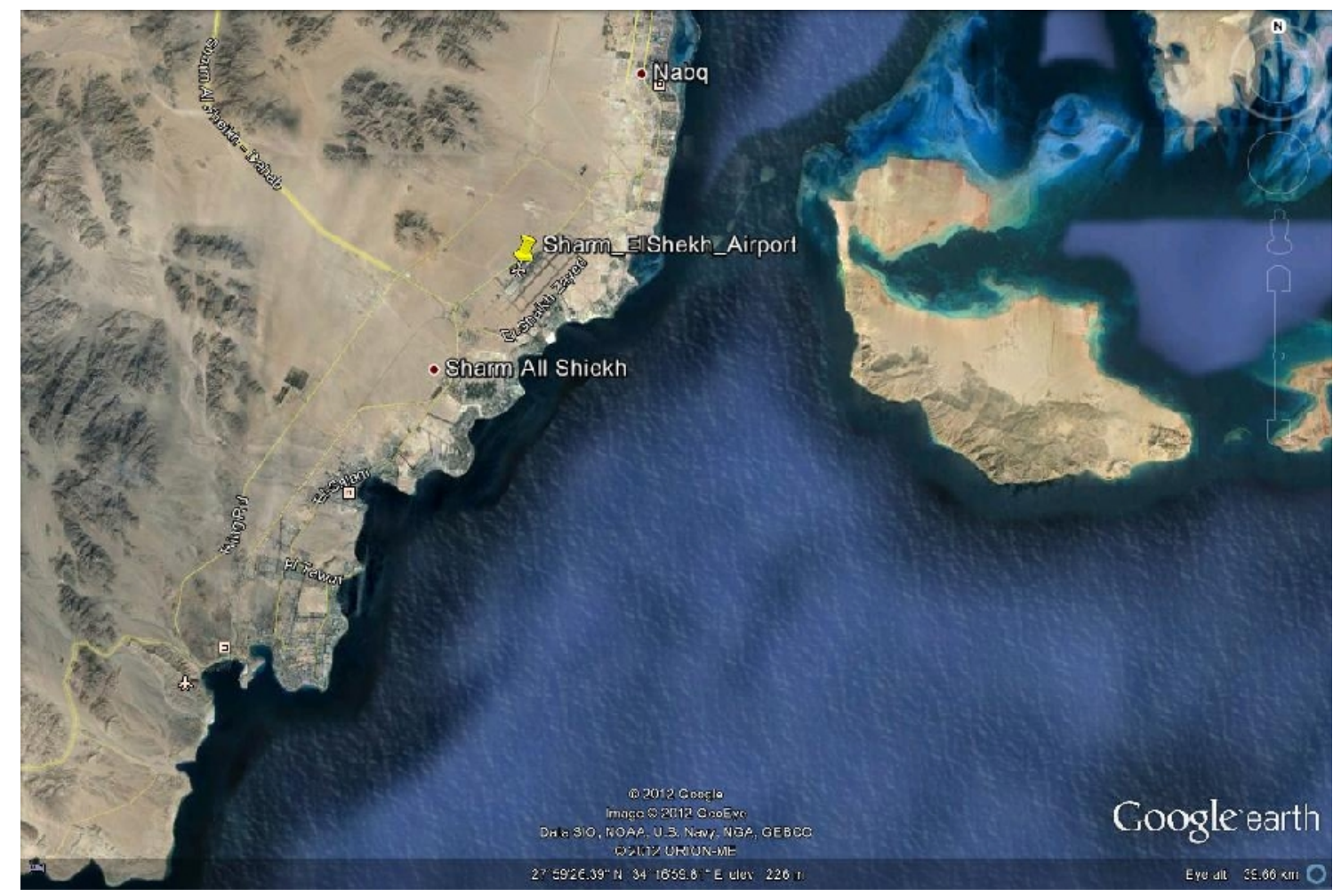

Figure (1): The Surrounding Terrain of Sharm El-shekh Airport

From deep investigation in Figure 2 and Figure 3, we can conclude the following:

- The coverage area increases with increasing the aircraft height which can be seen in the two figures and using the ITM and free space models. 
- The south coverage computed by the ITM results is larger than the free space model, at 100 meter, the free space model range is $57 \mathrm{~km}$ whereas it increases to $93 \mathrm{~km}$ which represents over $60 \%$ communication behind LOS range.

- The other directions coverage area is affected by area terrain. In the north direction, the coverage area is limited to few kilometers which can be claimed to the effect of Sinai mountains. Also, in a small portion of the south east directions, the coverage area is almost few kilometers which as a negative superposition of reflected, refracted and direct waves.

- On the other hand, the coverage increases to $115 \mathrm{~km}$ in the east directions which is almost $100 \%$ communication behind LOS range.

- Similar results can be observed from the case of 300 meter aircraft height given the LOS range is $87 \mathrm{~km}$, the maximum coverage range in south direction is $120 \mathrm{~km}(38 \%$ behind LOS communication range) and maximum overall coverage range is $145 \mathrm{~km}$ (67\% behind LOS communication range).

- The effect of terrains decreases as the aircraft height increases.

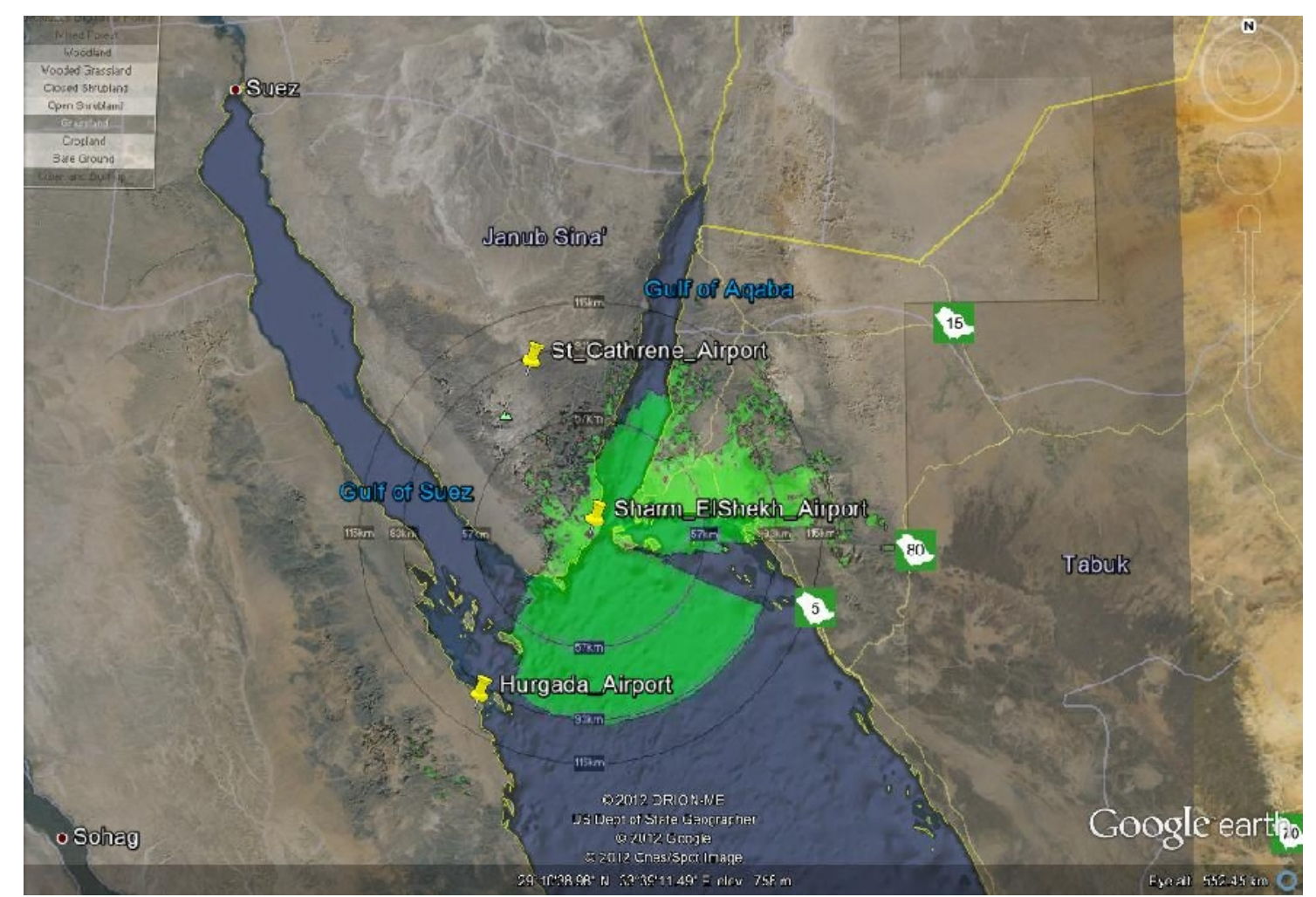

Figure (2): Prediction coverage area of Sharm El-shekh airport at 100 meter height 


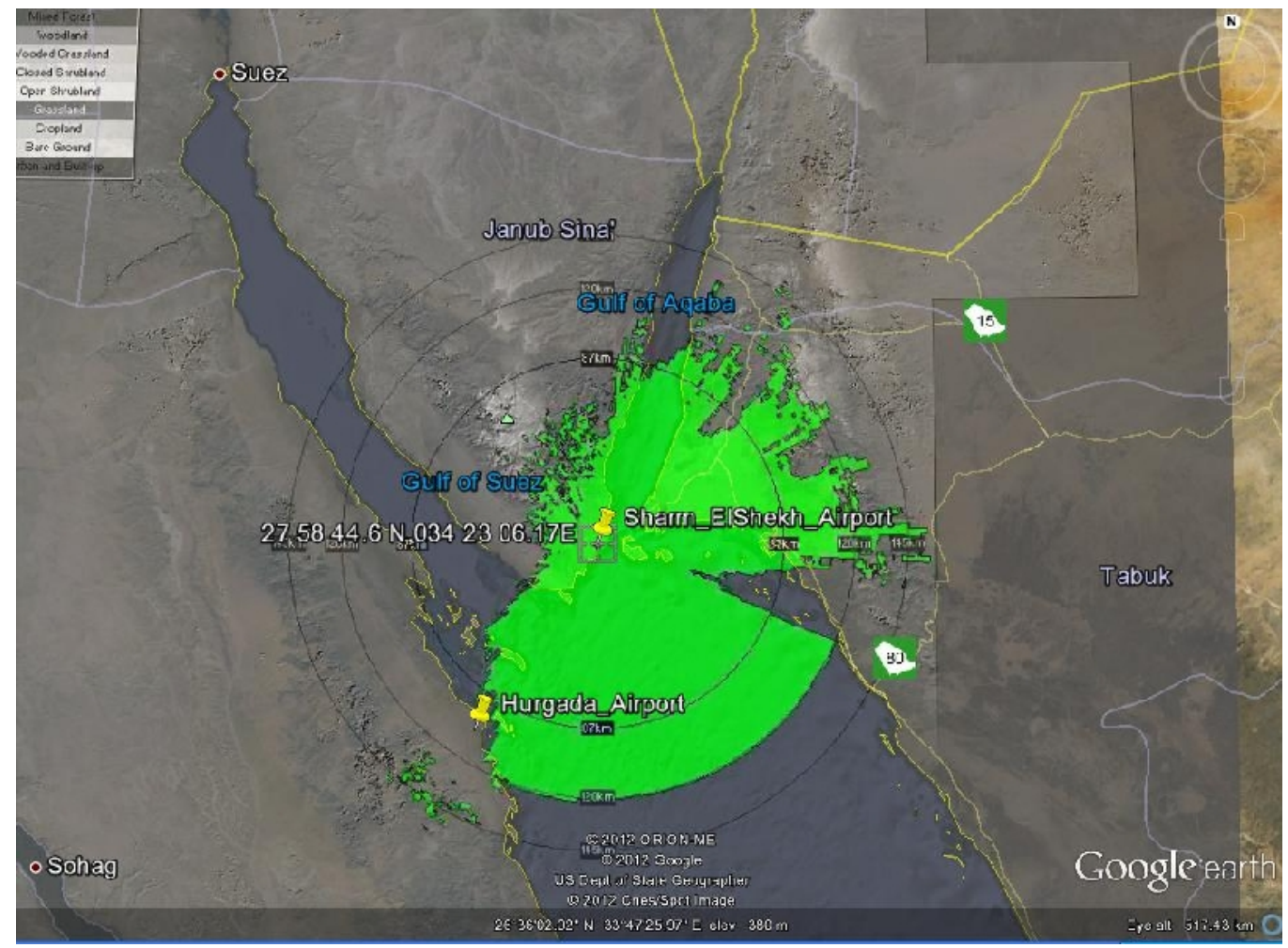

Figure (3): Prediction coverage area of Sharm El-shekh airport at 300 meter height B-Heavy Terrain Airport Results and Discussions

The coverage area of St Katherine airport is the second goal of this paper. The surrounding airport terrain is shown in Figure 4. As it can be observed, this surrounding terrain can be considered heavier terrains compared to the previous studied case. The Similar to Sharm El-shekh airport, the VOR system coverage area was designed assuming the aircraft heights of 100 meter and 300 meter.

The ITM and free space model results are computed and plotted in Figure 5 and Figure 6 for aircraft height of 100 and 300 meter, respectively. On contrast to previous studied case, the ITM computed coverage area at both aircraft height is less than the free space model. This can be claimed due to the effect of the very heavy irregular terrain of Sinai Mountains. Also, in both cases, the ITM computed coverage area can reach the Red sea which makes it very difficult for an aircraft to reach St. Katherine airport.

Combined electromagnetic coverage is a solution for this problem. Therefore, by reestimating the combined coverage of both Sharm El-Shekh and St Katherine airports as shown in Figure 7 and Figure 8 for aircraft height $=100$ meter and 300 meter, respectively. As shown in Figure 7, the combined two air port coverage does not succeed in overlapping with single St Katherine air port coverage. However, at aircraft height of 300 meter, the combined airports coverage demonstrates typical overlap between the single two airport overages. In other words, we can say by the aid of the two VOR systems at both airports, it is possible to guide an aircraft at low heights of 300 meter. 


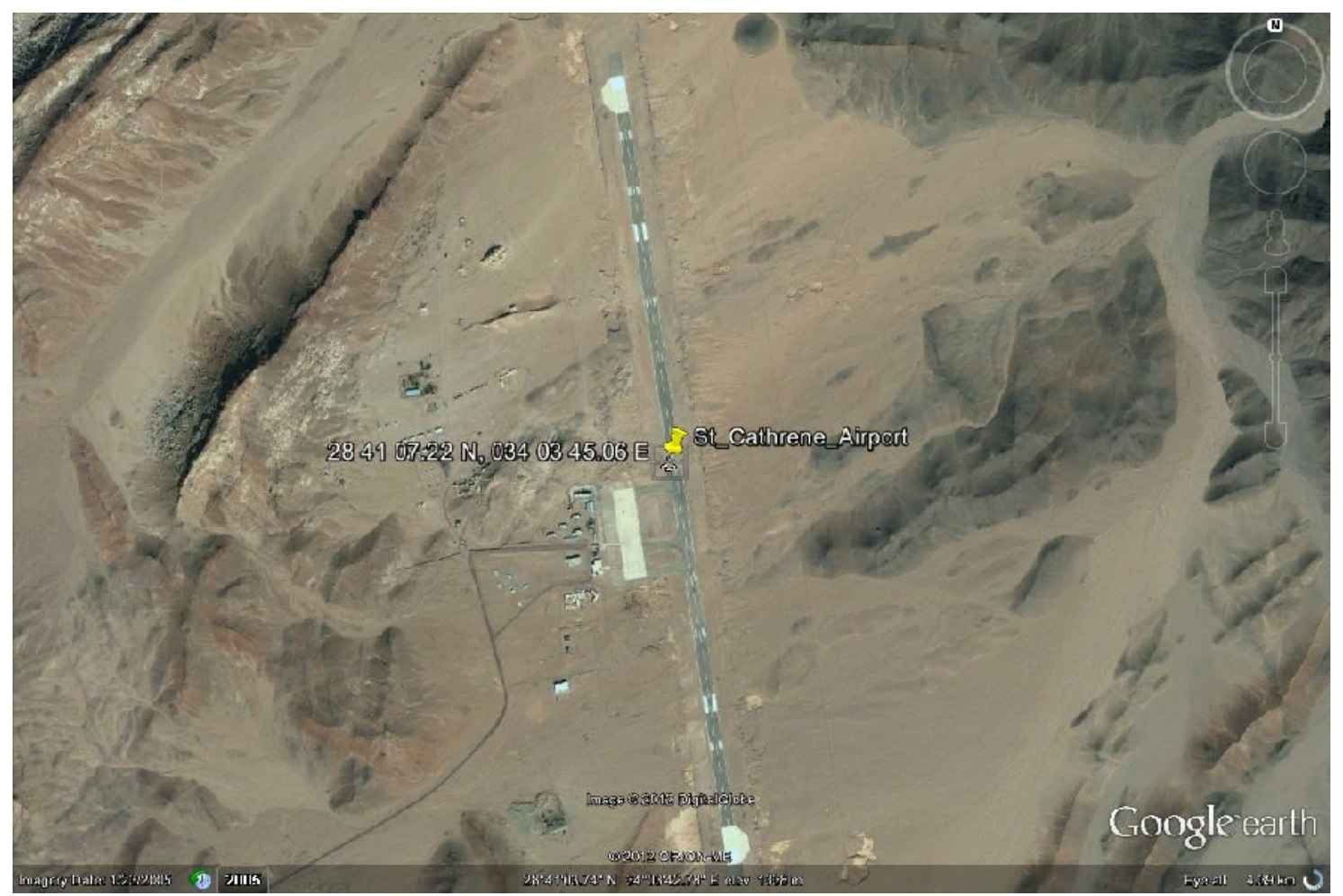

Figure (4): The Surrounding Terrain of St Katherine Airport

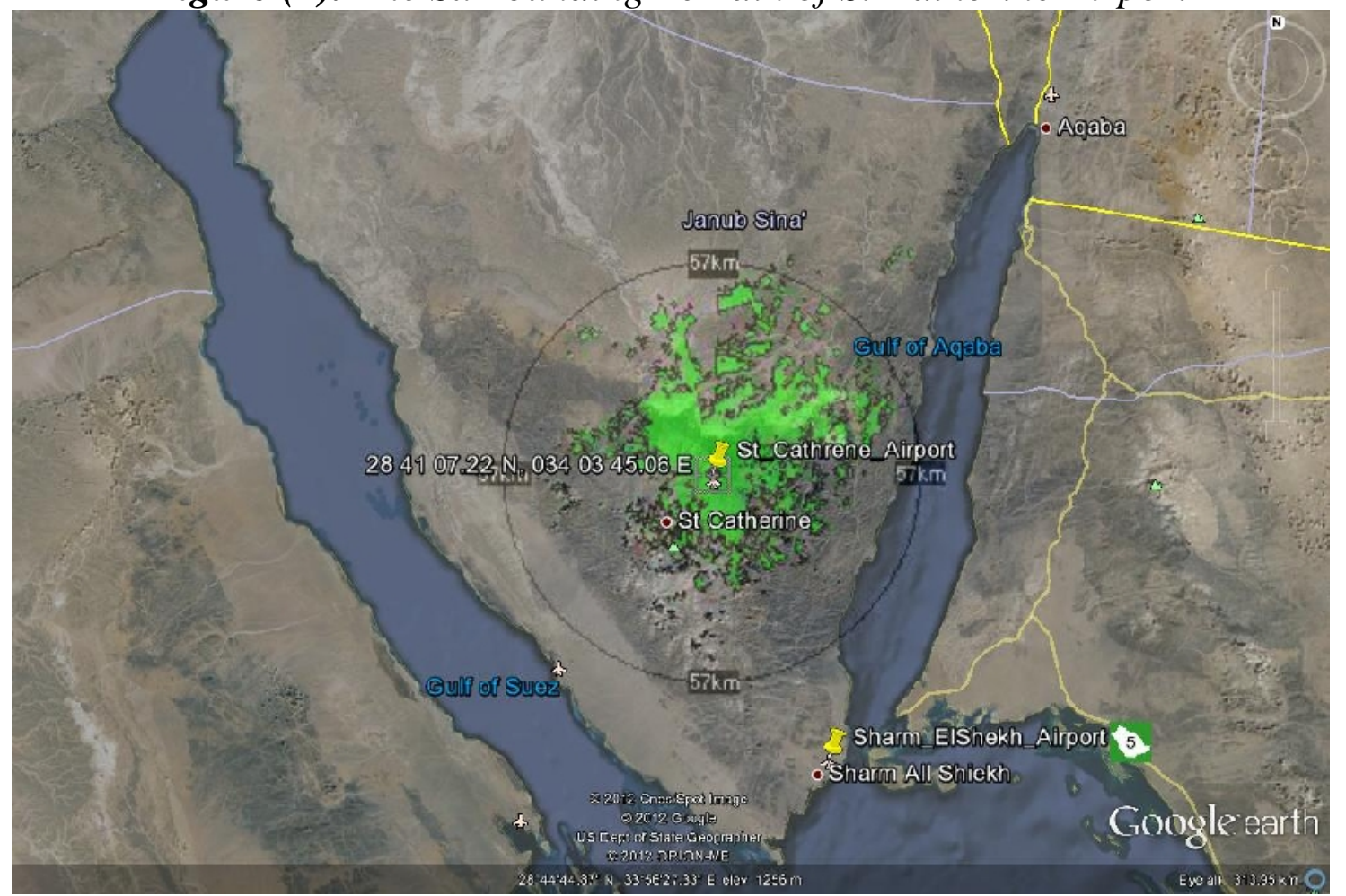

Figure (5): Prediction coverage area of St Katherine airport at 100 meter height 


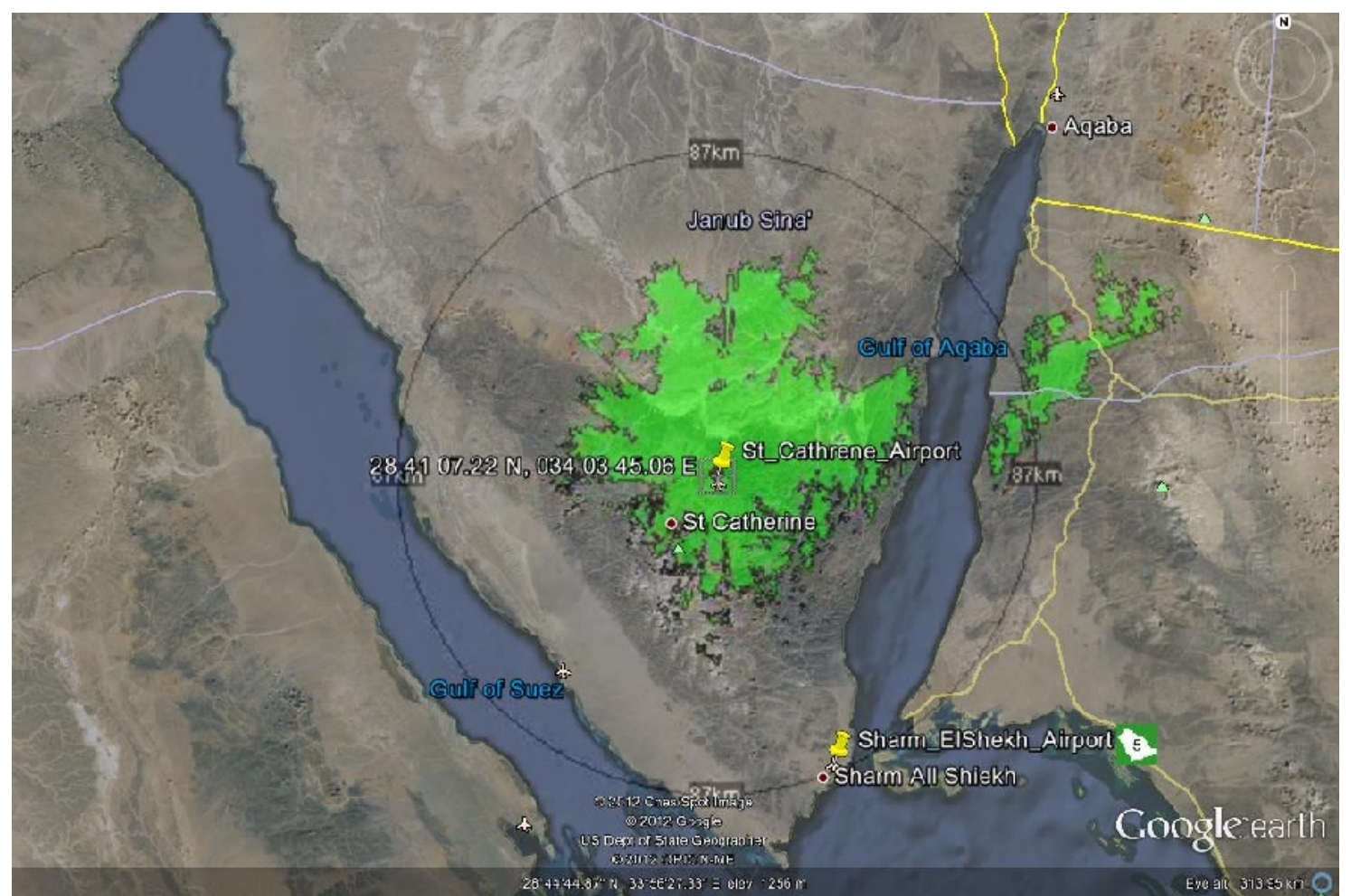

Figure (6): Prediction coverage area of St Katherine airport at 300 meter height

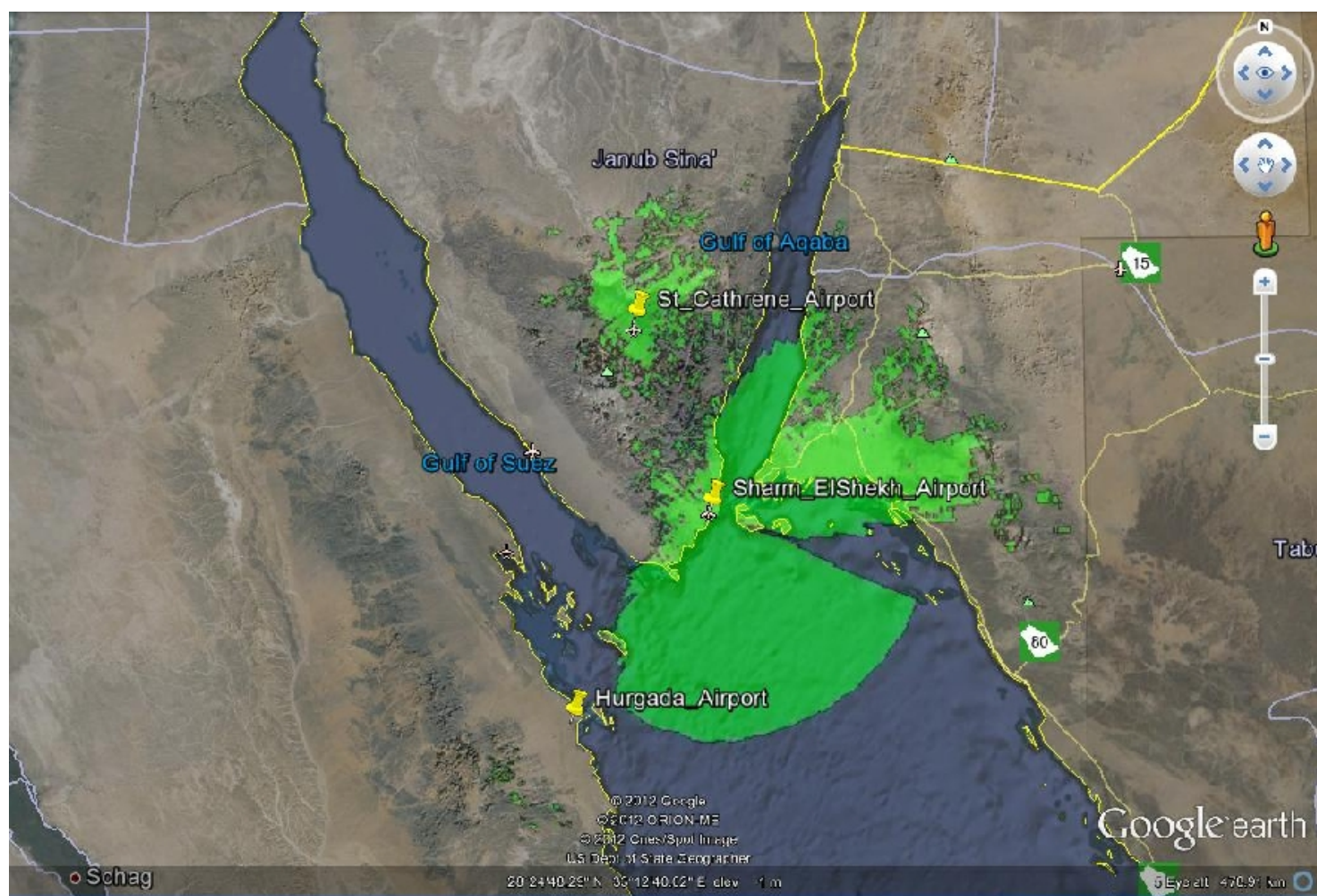

Figure (7): Prediction combined coverage area of Sharm El shekh and St Katherine airport at 100 meter height 


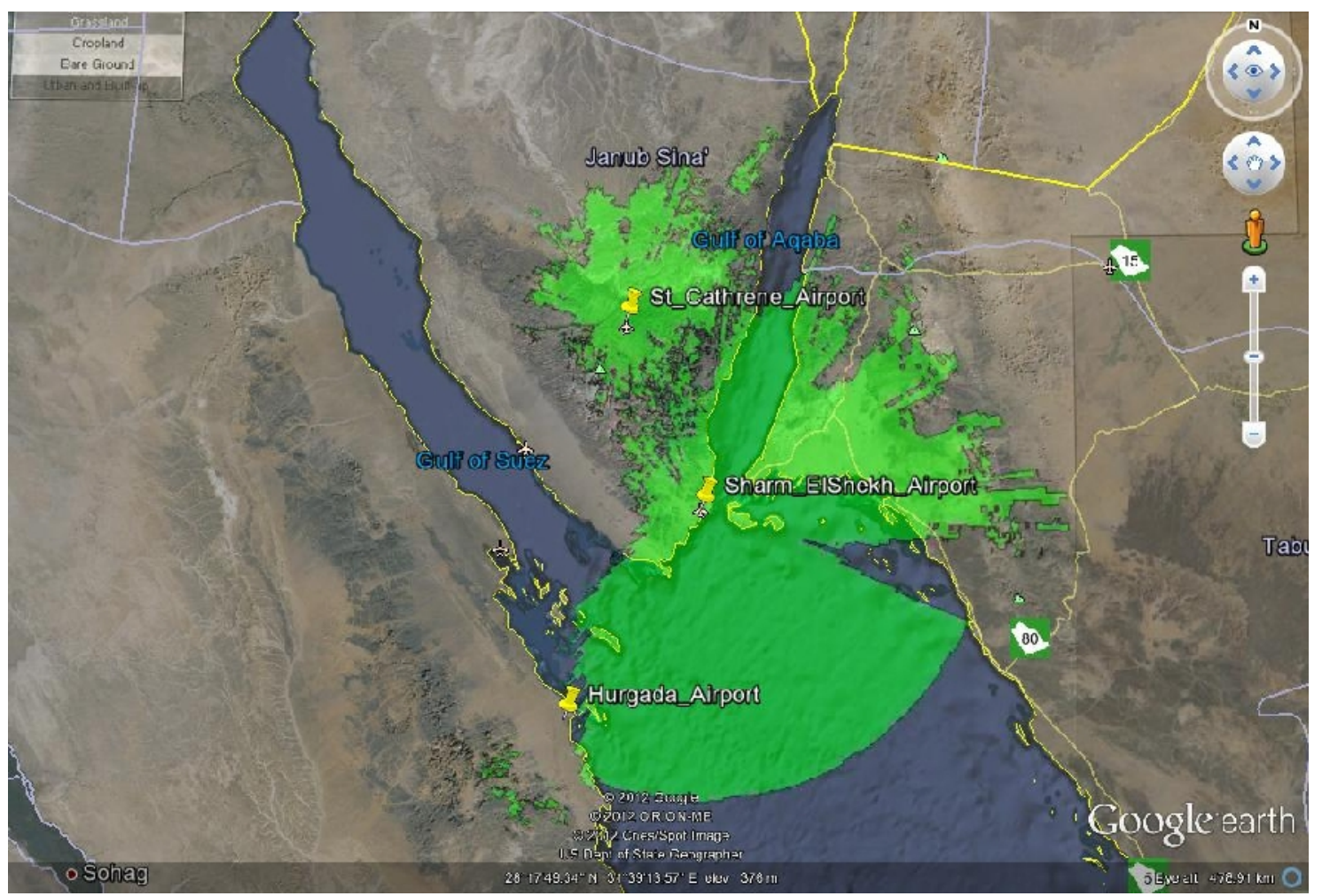

Figure (8): Prediction combined coverage area of Sharm El shekh and St Katherine

\section{Conclusions:}

airport at 300 meter height

This paper presents the single and combined coverage area of irregular terrain airports. The results were computed using the Longley Rice model and compared to simple free space model. The theoretical concepts of the employed model are discussed. Two different studied cases of medium terrain (Sharem El Shekh) and heavy terrain (St Katherine) air ports are introduced in details. The results confirm the behind of line of sight communication coverage by $60 \%$ for height of 100 meter at Sharem Elshekh air port and less than the line of sight communication range for St Katherine air port case.

\section{References:}

[1] G. Liang and H. L. Bertoni, "A new approach to 3-D ray tracing for propagation prediction in cities," IEEE Trans. Antennas Propagat., vol. 46, pp. 853-863, 1998.

[2] J. W. Ikegami, T. Takeuchi, and S. Yoshida, "Theoretical prediction of map field strength for urban mobile radio," IEEE Trans. Antennas Propagat., vol. 39, pp. 299302, 1991.

[3] J. W. McKown and R. L. Hamilton, "Ray tracing as a design tool for radio networks," IEEE Network Mag., vol. 5, no. 6, pp. 27-30, 1991. 
[4] K. R. Schaubach, N. J. Davis, and T. S. Rappaport, "A ray tracing method for predicting path loss and delay spread in microcellular environments," in Proc. IEEE Vehicular Technology Conf., 1992, pp. 932-935.

[5] V. Erceg, A. J. Rustako, and R. S. Roman, "Diffraction around corners and its effects on the microcell coverage area in urban and suburban environments at 900 MHz, 2 GHz, and 7 GHz," IEEE Trans Vehic. Technol., vol. 43, pp. 762-766, 1994.

[6] B. E. Gschwendtner, G. Wölfle, and F. M. Landsforfer, "Ray tracing versus ray launching in 3-D microcell modeling," in Proc. European Personal and Mobile Communications Conf., EPMCC'95, 1995, pp. 74-79.

[7]P. L. Rice, A. G. Longley, K. A. Norton, and A. P. Barsis, "Transmission loss predictions for tropospheric communication circuits," "Tech Note 101", U.S. Government Printing Office, Washington, DC, NBS Tech. Note 101, issued May 1965; revised May 1966 and Jan. 1967.

[8] A. G. Longley and P. L. Rice, "Prediction of Tropospheric radio transmission over irregular terrain, A Computer method-1968." ESSA Tech. Rep. ERL 79-ITS 67, U.S. Government Printing Office, Washington, DC, July 1968.

[9] G. A. Hufford, A. G. Longley, and W. A. Kissick, "A guide to the use of the ITS irregular terrain model in the area prediction mode," NTIA Repprt 82-100, Apr. 1982.

[10] Braemar Circle, William A Daniel, John E Dettra, Robert T Forrest, John P Murray, Neal H Shepherd, Floyd D Shipley, "Coverage Prediction for Mobile Radio Systems Operating in the 800/900 MHz Frequency Range," IEEE Trans. Vehicular. Technology, vol. VT-37, p. 3-72, 1988.

[11] M. Hata, "Empirical Formula for Propagation Loss in Land Mobile Radio Services", IEEE Transactions on Vehicular Technology, Vol, VT-29, No, 3, 1980

[12] FA. Isidoro, P,B, Cardoso, T.T. Guerra, L.M. Correia, "Adaption of the OkumuraHata Model to the City of Lisbon for short distances", COST 231 TD(95) 003, Bern, Switzerland, 18-21th January 1995.

[13] S. Kozono, K. Watanabe, "Influence of Environmental Buildings on UHF Land Mobile Radio Propagation", IEEE Transactions on Communications, Vol Com-25, No. 10,1977

[14] H. Kuschel, "Methoden zur Beriicksichtigung des Einflusses von Bewuchs und Bebauung auf Funkstreckenschatzungsdampfungen", Forschungsinstitut fiir Hochfrequenzphysik der FGAN e.V.

[15] U. Mollenbeck, "Erweiterung eines empirischen Wellenausbreitungsmodells durch Nahbereichsmodellierung in stadtischen Gebieten", Diplomarbeit an der RWTH Aachen, Lehrstuhl fiir Kommunikationsnetze, und der Mannesmann Mobilfunk GmbH, 1995 
[16] Y. Okumura et al., "Field Strength and its Variability in VHF and UHF Land Mobile Radio Service", Review of The Electrical Communication Laboratory, Vol 16, 1968.

[17] P. Schneider, F. Lambrecht, A. Baier, "Enhancement of the Okumura-Hata propagation model using detailed morphological and building data", In proc. Seventh IEEE International Symposium on Personal, Indoor and Mobile Radio Communications, 1996. PIMRC'96., Date of Conference: 15-18 Oct 1996, pp. 3438.

[18] Eugenia Montiel, Alberto S. Aguado, and François X. Sillion, "A Radiance Model for Predicting Radio Wave Propagation in Irregular Dense Urban Areas", IEEE Trans. On Ant. \& Prop., Vol 51, no. 11, Nov. 2003, pp. 3097-3108.

[19] O. Bendov, "On the validity of the LongleyRice $(50,90 / 10)$ propagation model for HDTV coverage and interference analysis," Proc. NAB Broadcast Engineering Conf., Las Vegas, NV, 1999.

[20] International Telecommunications Union (ITU), "VHF and UHF Propagation Curves for the Frequency Range from $30 \mathrm{MHz}$ to $1000 \mathrm{MHz}$," Rec. ITU-R P.370-7, 1995.

[21] C. Prez-Vega \& J.M. Zamanillo, "Path-loss model for broadcasting applications and outdoor communication systems in the VHF and UHF Bands," IEEE Trans. Broadcasting, Vol. 48, No. 2, Jun 2002, pp. 91-96. 